\title{
Nursing care of fragility fracture patients
}

DOI:

10.1016/j.injury.2018.06.036

\section{Document Version}

Accepted author manuscript

Link to publication record in Manchester Research Explorer

\section{Citation for published version (APA):}

Brent, L., Hommel, A., Maher, A. B., Hertz, K., Meehan, A. J., \& Santy-tomlinson, J. (2018). Nursing care of fragility fracture patients. Injury. https://doi.org/10.1016/j.injury.2018.06.036

\section{Published in:}

Injury

\section{Citing this paper}

Please note that where the full-text provided on Manchester Research Explorer is the Author Accepted Manuscript or Proof version this may differ from the final Published version. If citing, it is advised that you check and use the publisher's definitive version.

\section{General rights}

Copyright and moral rights for the publications made accessible in the Research Explorer are retained by the authors and/or other copyright owners and it is a condition of accessing publications that users recognise and abide by the legal requirements associated with these rights.

\section{Takedown policy}

If you believe that this document breaches copyright please refer to the University of Manchester's Takedown Procedures [http://man.ac.uk/04Y6Bo] or contact uml.scholarlycommunications@manchester.ac.uk providing relevant details, so we can investigate your claim.

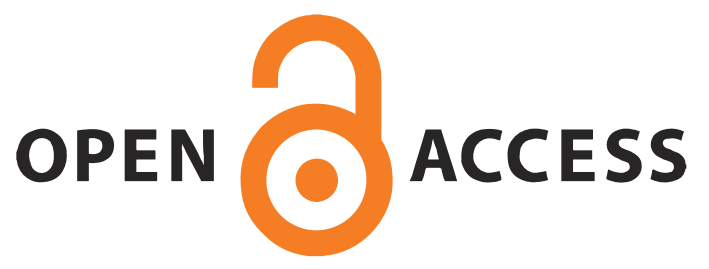




\section{Nursing care of fragility fracture patients}

CORRESPONDING AUTHOR: Louise Brent, MSc, BSc, RGN, RNP, Irish Hip Fracture Database and Major Trauma Audit Coordinator, National Office of Clinical Audit, RCSI, Dublin 2.

Affiliations: National Office of Clinical Audit, Royal College of Surgeons in Ireland, 123 St. Stephens Green, Dublin 2.

Correspondence: National Office of Clinical Audit, Royal College of Surgeons in Ireland, 123 St. Stephens Green, Dublin 2.

\section{Email: louisebrent@noca.ie}

Ann Butler Maher, MS, RN, FNP-BC (Retired), ICON Ambassador, Long Branch, NJ, United States of America

Karen Hertz, MSc, BSc, DPSN, RGN, Advanced Nurse Practitioner, University Hospital of North Midlands, NHS Trust, United Kingdom.

Ami Hommel, PhD, CNS, RN, Associate Professor, Malmö University, Malmo, Sweden, Skane University Hospital, Lund, Sweden

Anita J. Meehan, MSN, RN-BC, ONC, FNGNA, Clinical Nurse Specialist, Gerontology, Cleveland Clinic Akron General, Akron, Ohio, United States of America Julie Santy-Tomlinson, PhD, MSc, BSc, RGN, Senior Lecturer , Division of Nursing, Midwifery and Social Work, School of Health Sciences, Faculty of Biology, Medicine and Health, The University of Manchester

Keywords: nursing care, fragility fractures, orthopaedic nursing, geriatric nursing

\section{Abstract}

The challenge of caring for patients with fragility fractures is particularly acute for nursing teams who are in short supply and work with patients following fracture on a $24 \mathrm{~h}$ basis, coordinating as well as providing complex care. This paper considers the role of nurses within the orthogeriatric team and highlights the value of effective nursing care in patient outcomes. It explores the nature of nursing for patients with fragility fracture with a focus on the provision of safe and effective care and the coordination of care across the interdisciplinary team. It also highlights the need for specific skills in orthopaedic and geriatric nursing as well as specialist education.

\section{Introduction}


Patients with fragility fractures represent a significant challenge to the health care team. This challenge is particularly problematic for nursing teams who are struggling to provide adequate nursing care due to the global shortage of nurses, even in wealthier healthcare systems. Evidence based practise, improving standards and audit have all had a positive impact on the management of patients with fragility fractures. The nursing role in preventing morbidity and mortality and in ensuring patient safety during recovery and rehabilitation will be central to the future of continued improvement of fragility fracture care.

There is no single healthcare profession that can manage the care of fragility fracture patients in isolation, but there is encouraging evidence that patients' outcomes are improved if there is full collaboration across the many disciplines making up the 'orthogeriatric' team. This paper will focus on the impact of expert nursing care on patient outcomes while acknowledging that a fundamental characteristic of holistic care is that nurses must collaborate with others to achieve best practice [1].

The combined efforts of hip fracture audit, research and service improvement have resulted in significant improvements in hip fracture outcomes. There remains, however, significant mortality and morbidity that is more likely to be caused by care factors related to the quality of nursing care and the limitations of nursing resources than to the surgical event or medical care. Nursing has a significant role to play in the continued improvement in care standards and outcomes. It has been pointed out that surgery for hip fracture is now so well-crafted that its direct complications are now unusual and patients who struggle to recover now do so due to pre-existing problems such as frailty, cognitive and nutritional problems that worsen following surgery and can be significantly impacted on by effective nursing care [2].

\section{The nature of nursing}

Patients hospitalised because of a fragility fracture have numerous highly complex orthogeriatric care needs that need a team approach to care that includes skilled, compassionate nursing. The continued development and improvement of fragility fracture care requires nurses who have specific sets of both generic and specialist nursing skills, including those of both the orthopaedic nurse and the geriatric practitioner. Nurses spend the most time directly with patients and represent the largest group of healthcare professionals, so are in a central position to have a positive impact on outcomes. Even so, understanding the exact impact of nursing has been restricted by a lack of clarity regarding the complex role of the nurse and nursing's unique contribution to care over the century and a half since it became a formal profession.

One of the unique characteristics of nurses is that they spend the most time with patients, providing care across the 24-hour period, often over many days or weeks, in both primary and secondary care. They also have the greatest depth of relationships with the patient's family. Nurses' skills have a broad reach that includes; ensuring 
compassion, empathy and comfort are prevalent in care; coordinating care; undertaking clinical assessment; monitoring and minimising complications; providing education; primary and secondary fracture prevention and facilitating the team approach to rehabilitation. Planning and providing care that is holistic, patient-centred and seamless between hospital, community and other services involves a deep understanding of the recovery experience from the patient's perspective. Nursing teams in both secondary (hospital) and primary (community) settings work closely with families and other care providers to augment the experience of the fracture, surgery, recovery and subsequent care through their close relationships with everyone involved. In some localities, nurses now work in diverse, autonomous and highly skilled roles including; nurse specialists, advanced nurse practitioners and consultant nurses, offering high quality, age-sensitive care tailored to the needs of older people with fragility fractures [3].

The two central purposes of nursing are: 1) The provision of safe and effective care and; 2) Coordination of the multidisciplinary team. The International Council of Nursing (ICN) [4] described nursing as: "...encompassing collaborative and autonomous care of all individuals regardless of age, including the provision of health promotion, prevention of illness and the care of sick and dying patients". Due to the extended time nurses spend with patients and their families, they gain in-depth appreciation of the patient's complex needs. Through this intimate knowledge of the patient, nurses can provide leadership and coordination of the team which enables them to significantly influence care pathways and service development.

\section{Provision of safe and effective care}

Fragility fractures commonly result is a reduced level of functional ability and patients rarely regain their full pre-fracture level. Regardless of the clinical environment in which fragility fracture patients are managed, nurses need to possess a common set of core skills to provide safe and effective care for patients with complex needs. Recognising the fact that providing care to older patients following fragility fracture is challenging and complex is a critical step in facilitating positive outcomes. Hip fracture is known to carry a high incidence of morbidity, disability and mortality of $10 \%$ at one month and $30 \%$ at one year [5], posing a challenge to nurses in managing complex multifactorial issues relating to advanced patient age, frailty, pre-existing comorbidities, sarcopenia, reduced physical reserves and cognitive impairment.

Nurse-sensitive patient outcomes such as patient comfort and quality of life, risk outcomes and safety, patient empowerment and patient satisfaction are traditionally viewed as indicators of the quality of nursing care [6]. It is more likely, however, that these parameters reflect the care provided by the whole team. Indicators that are more specific to the generic nursing role include; healthcare-associated infection, pressure ulcers, falls, drug administration errors and patient satisfaction [7, 8]. There is a need to develop more advanced nurse sensitive indicators, for example, for: pain, delirium, pressure ulcers, hydration and nutrition, constipation, prevention of secondary 
infections and venous thromboembolism (VTE). Indicators will need to take account of evidenced-based nurse management strategies that co-exist with medical models of care; reducing the risk of developing complications, aiming to reduce the risk of morbidity and mortality, whilst improving recovery, maintaining functional ability and improving patient outcomes and experiences [3]. Demonstrating such impact will enable nurses to establish the true value of their input and to argue for the increased resources necessary for continued care improvement. Hip fracture audit and other measures of quality of fragility fracture care must capture aspects of the patient's care which can be influenced by nursing. The development of nurse-sensitive indicators that coexist with medical parameters relating to pain, delirium, pressure ulcers, hydration, nutrition, constipation, prevention of secondary infections and venous thromboembolism (VTE) are most likely to capture the nursing contribution and lead to care improvement $[9,10]$. Data about these parameters needs to be collected along with other information that is more difficult to elucidate such as the patient experience, understanding and perceptions about their own goals and the impact of care.

The maintenance and restoration of the functional capacity of patients with fragility fractures is a primary goal for the whole interdisciplinary team, but nursing interventions have a major influence [11]. Bed rest and immobility have a significant impact on older people, leading to loss of muscle function and strength, less efficient respiration and increased risk of respiratory infections, pressure injuries and orthostatic hypotension. These influences further weaken bone structure, increase risk of fractures and impact on psychological issues such as lack of motivation.

Early mobility reduces the risk of harmful events and enables better functional recovery and independence. Nursing teams work particularly closely with therapists in ensuring that immobility lasts the shortest time possible and that the recovery of mobility is commenced as soon as the patient is able. Because of the $24 \mathrm{~h}$ nature of nursing in the hospital setting it is the nursing team, however, who are most inextricably linked to patient progress towards recovery of mobility as the practice for this takes place during usual activities of daily living such as, for example, while the patient is being supported moving from bed to chair and walking to the toilet. Pain management, nutrition, hydration, remobilisation, rehabilitation and motivation are all central to prevention of complications for patients following hip fracture and that these are all nursing care priorities. Nurses and the care staff with whom they work are also key observers of patients. Knowing how to recognise signs of complications such as delirium, pressure injuries and venous thromboembolism and is central to ensuing early intervention is achieved and causative factors are reversed.

Pressure injuries (formerly known as pressure ulcers/sores) are one of the most important nurse-sensitive indicators of quality care and are considered in many national hip fracture registries/databases. In a pan-European study of hospitalised hip fracture patients, Lindholm et al. [12] reported that there was a $10 \%$ pressure injury rate recorded on admission and that this increased by more than double to $22 \%$ at discharge. In a Canadian study, the rate was significantly higher, at $16-55 \%$ [13]. The 
most important package of evidence-based strategies for pressure injury prevention are enshrined in international guidelines [14] and include: assessment and documentation of skin state and assessment of pressure ulcer risk using a validated tool; timely, detailed documentation that includes correct staging of any pressure injuries; nutritional assessment and intervention; consideration of pressure relieving and reducing bed and chair surfaces, regular repositioning; and early supported mobilisation. Pressure injury prevention involves an understanding of precipitating factors and a complex interplay of interventions that require a sustained, consistent coordinated approach to care that encompasses all other aspects of nursing such as mobilisation, pain management, skin care, continence care, nutrition and hydration illustrating. This illustrates the complexity of nursing care that is difficult to capture and difficult to measure [2].

The availability of enough appropriately educated and skilled nurses to provide this fundamental care is a constant source of concern. In a large international study, Aiken et al [15] demonstrated that an increase in a nurses' workload by one patient, from eight to nine patients per qualified nurse, increased the likelihood of an inpatient dying within 30 days of admission by $7 \%$. One explanation for this is that under-resourcing nursing teams means that there is insufficient capacity for the team to undertake actions to prevent morbidity and mortality. Studies have indicated that this 'missed care' is associated with one or more adverse patient outcomes including; medication errors, urinary-tract infections, falls, pressure injuries, critical incidents, quality of care and patient readmissions. The quality of studies relating to missed care is, however, weak and the link between missed care and mortality is uncertain [16].

The causes and risk factors for falls and fractures are complex. Each fall and fracture should be seen by the nursing and interdisciplinary team as a collaborative opportunity to prevent the next fall or fracture. The nursing role in achieving this aim is central to its success. Nurses are perfectly placed to assess the level of fall risk through patient observation and to initiate and co-ordinate the multi-disciplinary effort in preventing further falls and managing osteoporosis. Ongoing investigation of the cause of the presenting fall that led to the fracture should be conducted collaboratively [17]. Just as nurses provide advice to patients about smoking cessation or obesity, they should also be equipped and empowered to discuss with patients and families the strategies that need to be in place to prevent further falls and fractures. Nurses must be enabled, through education and training, to use the time they spend giving care with patients and families to educate them about the cause of fragility fractures and importance of bone health. The opportunity to impress upon the patient the importance of taking bone protection medication while still very much impeded by the fracture could influence the level of compliance with osteoporosis medication and advice after discharge.

\section{Coordination of care across the interdisciplinary team}


It is a fundamental characteristic of holistic care that nurses are required to collaborate with others in order to achieve best practice [1]. The challenge for nurses when working within the orthogeriatric team is that, in order to access acute fracture care, the patient inevitably requires care in a variety of hospital departments including units such as emergency departments, fracture clinics and trauma wards. These are care settings that have historically evolved to treat all adult patients, irrespective of age, and following all types of musculoskeletal injury. This fails to recognise the specific and complex needs of patients who have sustained a fracture, are older, frail and have significant co-morbidity.

The sharing of care between orthopaedic surgeons and orthogeriatricians would become fragmented and less effective if the care is not managed or coordinated effectively. Nurses are an integral contributor to the orthogeriatric team within the 'The Hip Fracture Programme' [18] because of their role as care co-ordinators [19]. Coordination is usually provided by nurses working in general orthopaedic trauma units. This is often led by a specialist nurse or designated coordinator, depending on the size, culture and organisation of each individual unit. Such roles may include; hip fracture nurse specialists, elderly/elder care nurse specialists, trauma nurse coordinators, nurse practitioners and advanced nurse practitioners. The true value of such roles, however, has yet to be formally evaluated.

The role of the nurse in coordination may vary according to the needs and culture of the organisation and may fluctuate between regular team orthogeriatric ward rounds, daily ward rounds, team discussions relating to discharge co-ordination and coordination of an interdisciplinary hip fracture programme. The main issue is that nurses, who are permanently based on the unit, have a close professional relationship with patient as well as the other members of the orthogeriatric team. They are the natural link between all other team members, patients and families or care givers.

\section{Orthogeriatric nursing education}

It is argued that nursing care is provided within a professional framework that includes professional standards and internal regulation and within a culture of constant skills and knowledge development through learning and reflection. Many nurses working in orthogeriatric settings, however, are more likely to have been educated solely in relation to the care of adults with musculoskeletal problems rather than to meet the complex needs of older people. Nurses working with patients with fragility fractures need multiple 'orthogeriatric' specialist skills that combine orthopaedic care expertise with that of the care of older people. With this comes the need for an in-depth knowledge of the theory underpinning care. Few nurses working with patients with fragility fractures have undertaken education beyond that of their initial nursing qualification and must rely on knowledge and skills developed through generic, rather than specialist, education. Consequently, there is, an important education and skills gap that can result in care which does not meet all needs. 
Competence is a defining feature of any professional practise [20] but can only be achieved if nurses have the requisite knowledge and skills to deliver care effectively to specific groups of patients. It has been demonstrated that care provided by nurses with a graduate education has a positive impact on patient mortality in general hospital settings [15]. The benefits of providing highly skilled and specialist nursing of fragility fracture patients by practitioners with advanced, specific education has never been explored, but it is likely that the development of specialist orthogeriatric nursing education could have a positive impact on patient outcomes.

\section{Conclusion}

There is a global shortage of nurses due to changing demographics, political ideologies for health care and education and resourcing problems. Unless the nursing resource is protected and grows, the added nursing value needed to continue to improve outcomes and the quality of care for patients with fragility fractures will not happen. Sahota \& Currie [21] point out that: "...looking after hip fracture patients well is a lot cheaper than looking after them badly", and it may well be the nursing resource that is significant in this, meaning that it may be necessary for the multidisciplinary community, through organisations such as the Fragility Fracture Network (FFN) [22] who could lobby governments and health and social care providers of to ensure that nurses are able to do undertake their role effectively.

Nurses play a central role in the coordination, provision and monitoring of care for patients with fragility fractures with far reaching benefits long after discharge. The evolution of specialist nursing roles has seen autonomous practitioners very capably manage cohorts of fragility fracture patients, but the care elements discussed in this paper should be within every nurse's competence when providing care for patients with fragility fractures. The landscape of healthcare is changing and the reality is that nurses will be facing an increasingly older cohort of patients at a time when the nursing resource is under political and financial pressure. Many of the elements discussed here would apply to many other groups of older patients. It is essential, therefore, to raise the priority of caring for older patients in the training and education of new and existing nurses and to champion increases in the available nursing resource.

\section{References}

1. Hall C, Ritchie D. What is Nursing? Exploring Theory and Practice. 3rd Edn 2013; London: Sage/Learning Matters

2. Johansen A, Boulton C, Hertz K, Ellise M, Burgon, Rai S, Wakeman R. The National Hip Fracture Database (NHFD) - Using a national clinical audit to raise standards of nursing care. International Journal of Orthopaedic and Trauma Nursing. 2017;26:3-6 
3. Hertz K, Santy-Tomlinson J. The Nursing Role. In: Falaschi, P. Marsh, D. Orthogeriatrics. 2017; 131-144 Springer: Switzerland.

4. International Council of Nurses (ICN) Definition of Nursing. 2010; Geneva: ICN http://www.icn.ch/who-we-are/icn-definition-of-nursing/

5. Lisk R, Yeong K. Reducing mortality from hip fractures: a systematic quality improvement programme BMJ Open Quality. 2014; doi:

10.1136/bmjquality.u205006.w2103

6. Dubois C-A, D'Amour D, Pomey M.-P, Girard F, Brault I. Conceptualizing performance of nursing care as a prerequisite for better measurement: a systematic and interpretive review. BMC Nursing 2013;12(7):12-7

7. Heslop L, Liu S. Nursing-sensitive indicators: a concept analysis. Journal of Advanced Nursing. 2014; 70(11):2469-2482.

8. Maben J, Morrow E, Ball J, Robert G, Griffiths P. High Quality Care Metrics for Nursing. 2012 National Nursing Research Unit, King's College London http://eprints.soton.ac.uk/346019/1/High-Quality-Care-Metrics-for-Nursing----Nov2012.pdf

9. Maher AB, Meehan AJ, Hertz K, Hommel A, MacDonald V, O'Sullivan M, Specht $\mathrm{K}$, Taylor $\mathrm{A}$. Acute nursing care of the older adult with fragility hip fracture: an international perspective. Part 1. International Journal of Orthopaedic and Trauma Nursing. 2012;16(4):177-194

10. Maher AB, Meehan AJ, Hertz K, Hommel A, MacDonald V, O'Sullivan M, Specht $\mathrm{K}$, Taylor $\mathrm{A}$. Acute nursing care of the older adult with fragility hip fracture: an international perspective. Part 2. International Journal of Orthopaedic and Trauma Nursing. 2013;17(1);4-18

11. Brent L. Coffrey A. Patient's perceptions of their readiness for discharge following hip fracture surgery. International Journal of Orthopaedic and Trauma Nursing 2013;17:190-198

12. Lindholm C, Sterner E, Romanelli M, Pina E, Torray Bou J, Hitenen H, livananien A, Gunningberg, Hommel A, Klang B, Dealey C. Hip fracture and pressure ulcers the Pan-European pressure ulcer study - intrinsic and extrinsic risk factors. International Wound Journal. 2008;5(2);315-328

13. Campbell KE, Woodbury G, Houghton P. Heel pressure ulcers in orthopaedic patients: a prospective study of incidence and risk factors in an acute care hospital. Ostomy Wound Management, 2010;56(2);44-45

14. National Pressure Ulcer Advisory Panel, European Pressure Ulcer Advisory Panel and Pan Pacific Pressure Injury Alliance. Prevention and Treatment of Pressure Ulcers. Emily Haesler (Ed.). Cambridge Media: Perth, Australia; 2014. 
15. Aiken L, Sloane D, Bryneel L, Van den Heede K, Griffiths P, Busse R, Diomidous M, Kinnunen J. Kozka M, Lasaffre E, McHugh M, Moreno-Casbas M, Rafferty A, Schwendimann, Scott $P$, Tishelman C, van Achterberg T, Sermeus W. (Nurse staffing and education and hospital mortality in nine European countries: a retrospective observational study. Lancet 2014;383(993);1824-1830 http://dx.doi.org/10.1016/S0140-6736(13)62631-8

16. Recio-Saucedo A, Dall'Ora C, Maruotti A, Ball J, Briggs J, Meredith P, Redfern $O$, Kovacs C, Prytherch D, Smith G, Griffiths P. What impact does nursing care left undone have on patient outcomes? Review of the literature. Journal of Clinical Nursing. 2017 doi: 10.1111/jocn.14058 9 (Epub ahead of print)

17. Martin FC. Frailty, sarcopenia, falls and fractures. In: Falaschi, P. \& Marsh, D. Orthogeriatrics. 2017; 47-62 Springer: Switzerland

18. Meehan AJ, Hommel A, Hertz K, MacDonald V, Maher A. Care of the older adult with fragility hip fracture. In: Boltz, M., Capezuti, E. Fulmer, T. Zwicker, D. (Eds) Evidence-based Geriatric Nursing Protocols for Best Practice. 2016; Springer.

19. National Institute for Health and Care Excellence (NICE) The management of hip fracture in adults. CG 124. 2011

https://www.nice.org.uk/guidance/cg124/resources/hip-fracture-management35109449902789

20. Drozd, M. Jester, R. Santy, J. The inherent components of the orthopaedic nursing role: an exploratory study. Journal of Orthopaedic Nursing 2007:11(1);43-52

21. Sahota, O. Currie, C. Hip fracture care: all change. Editorial. Age and Ageing. 2008:37; $128-129$

22. Fragility Fracture Network (FFN) http://fragilityfracturenetwork.org/ 